\title{
Measuring the atomic scattering factors near the iridium L-edges for the Athena silicon pore optics reflector
}

\author{
Hisamitsu Awaki, ${ }^{\text {a,* }}$ Yoshitomo Maeda, ${ }^{\text {b }}$ Hironori Matsumoto, \\ Sara Svendsen $\odot{ }^{\mathrm{d}}$ Marcos Bavdaz, ${ }^{\mathrm{e}}$ Maximilien Collon $\odot,{ }^{\mathrm{f}}$ \\ Kazunori Asakura, ${ }^{c}$ Finn E. Christensen, ${ }^{d}$ Desiree D. M. Ferreira ${ }^{\circ},{ }^{d}$ \\ Matteo Guainazzi, ${ }^{\mathrm{e}}$ Masato Hoshino, ${ }^{\mathrm{g}}$ Shuntaro Ide, ${ }^{\mathrm{c}}$ Kazunori Ishibashi, ${ }^{\mathrm{h}}$ \\ Wansu Kan, ${ }^{a}$ Sonny Massahi $\odot,{ }^{d}$ Takuya Miyazawa, ${ }^{i}$ Sadayuki Shimizu, \\ Brian Shortt, ${ }^{\mathrm{e}}$ Yusuke Takehara, ${ }^{\mathrm{h}}$ Keisuke Tamura, ${ }^{\mathrm{j}}$ Kentaro Uesugi,g \\ Richard Willingale, ${ }^{\mathrm{k}}$ Tomokage Yoneyama, ${ }^{\mathrm{c}}$ and Atsushi Yoshidah \\ ${ }^{a}$ Ehime University, Faculty of Science, Matsuyama, Japan \\ ${ }^{\mathrm{b}}$ Institute of Space and Astronautical Science, Japan Aerospace Exploration Agency, \\ Sagamihara, Japan \\ 'Osaka University, Graduate School of Science, Osaka, Japan \\ ${ }^{\mathrm{d}}$ Technical University of Denmark, DTU Space, National Space Institute, Lyngby, Denmark \\ ${ }^{e}$ European Space Research and Technology Centre, Noordwijk, The Netherlands \\ f cosine, Warmond, The Netherlands \\ ${ }^{\mathrm{g} J a p a n ~ S y n c h r o t r o n ~ R a d i a t i o n ~ R e s e a r c h ~ I n s t i t u t e / S P r i n g-8, ~ S a y o, ~ J a p a n ~}$ \\ ${ }^{\mathrm{h}}$ Nagoya University, Graduate School of Science, Nagoya, Japan \\ iOkinawa Institute of Science and Technology Graduate University, Okinawa, Japan \\ ${ }^{\mathrm{j}}$ NASA Goddard Space Flight Center, Greenbelt, Maryland, United States \\ ${ }^{\mathrm{k}}$ University of Leicester, Department of Physics and Astronomy, Leicester, United Kingdom
}

\begin{abstract}
Athena, a future high-energy mission, is expected to consist of a large aperture x-ray mirror with a focal length of $12 \mathrm{~m}$. The mirror surface is to be coated with iridium and a low $Z$ overcoat. To define the effective area of the $\mathrm{x}$-ray telescope, the atomic scattering factors of iridium with an energy resolution less than that $(2.5 \mathrm{eV})$ of the $\mathrm{x}$-ray integral field unit are needed. We measured the reflectance of the silicon pore optics mirror plate coated with iridium in the energy range of 9 to $15 \mathrm{keV}$ and that near the iridium L-edges in steps of 10 and $1.5 \mathrm{eV}$, respectively, at the synchrotron beamline SPring-8. The L3, L2, and L1 edges were clearly detected around 11,215, 12,824 , and $13,428 \mathrm{eV}$, respectively. The measured scattering factors were $\sim 3 \%$ smaller than the corresponding values reported by Henke et al., likely due to the presence of an overlayer on the iridium coating, and were consistent with those measured by Graessle et al. The angular dependence of the reflectivity measured indicates that the iridium surface was extremely smooth, with a surface roughness of $0.3 \mathrm{~nm}$. (C) The Authors. Published by SPIE under a Creative Commons Attribution 4.0 Unported License. Distribution or reproduction of this work in whole or in part requires full attribution of the original publication, including its DOI. [DOI: 10.1117/1.JATIS.7.1.014001]
\end{abstract}

Keywords: x-ray optics; Athena; silicon pore optics; atomic scattering factors; iridium.

Paper 20119 received Aug. 4, 2020; accepted for publication Dec. 16, 2020; published online Jan. 6, 2021.

\section{Introduction}

The Advanced Telescope for High-Energy Astrophysics (Athena) ${ }^{1}$ is a next-generation x-ray telescope to be developed by the European Space Agency (ESA) as a part of its Cosmic Vision L2 program. Athena is expected to operate in the energy range of 0.2 to $12 \mathrm{keV}$ and realize the advanced x-ray imaging and spectroscopy of the hot Universe. The key scientific objectives are to determine how and when large-scale hot gas structures formed in the Universe, to perform a complete census of black hole growth in the Universe, and explore high-energy phenomena, which are expected to provide a valuable basis for astrophysics research in the 2030s.

*Address all correspondence to Hisamitsu Awaki, awaki@astro.phys.sci.ehime-u.ac.jp 
Athena is expected to consist of a large aperture $\mathrm{x}$-ray mirror ${ }^{2}$ with a focal length of $12 \mathrm{~m}$ and a goal of an on-axis angular resolution of 5 arc sec half energy width (HEW). The focal plane contains two instruments: a wide field imager ${ }^{3}$ and an $\mathrm{x}$-ray integral field unit (X-IFU). ${ }^{4}$ In the design of the large aperture telescope, a considerably high ratio of the collecting area to the mass is realized by employing ESA's silicon pore optics (SPO) technology. This SPO telescope adopts Wolter-I type grazing optics, ${ }^{5}$ and in the current baseline coating design, the reflector surface is expected to be coated with iridium. The large aperture telescope will be composed of $\sim 700$ SPO modules, with each mirror module containing 70 mirror plate pairs. ${ }^{2}$ The effective area of the Athena telescope is to be determined by summing the geometrical area times the individual reflectance of each module based on the telescope design. Specifically, to define the effective area in the high-energy region, it is necessary to ensure a suitable reflectance near the iridium Ledges. Because the X-IFU is designed to realize spatially resolved high-resolution spectroscopy with an energy resolution of $2.5 \mathrm{eV},{ }^{4}$ the reflectance should be determined with an energy pitch of $<2.5 \mathrm{eV}$ to suitably define the effective area. Henke et al. ${ }^{6}$ determined the atomic scattering factors for various elements at an energy range of 10 to $30,000 \mathrm{eV}$. However, Henke et al.'s data ${ }^{6}$ were based on the empirical cross section by Biggs and Lighthill ${ }^{7}$ above $10 \mathrm{keV}$. Thus, the optical constants of iridium near L-edges are not reproduced in the energy resolution of the transition edge sensor (TES) of the X-IFU. Therefore, it is necessary to measure the reflectance of the SPO mirror with an energy pitch smaller than the spectral resolution of the X-IFU.

In the previous work, ground calibration was performed, and the x-ray properties of $\mathrm{x}$-ray mirrors were clarified. Specifically, iridium optical constants for the Chandra $\mathrm{x}$-ray observatory were measured in the energy band from 50 to $12000 \mathrm{eV}^{8}$ to tabulate the iridium optical constants over the full range for this observatory. Kikuchi et al. ${ }^{9}$ measured the atomic scattering factors around the gold L-edges for the ASTRO-H (Hitomi) ${ }^{10}$ telescope with a sub-eV energy pitch matching the spectral resolution $(\sim 5 \mathrm{eV})$ of the microcalorimeter soft $\mathrm{x}$-ray spectrometer. ${ }^{11,12}$ To identify the x-ray property of the SPO mirror covering the energy range of Athena, we attempted to perform the x-ray measurements in the high-energy region from 9000 to $15,000 \mathrm{eV}$ at the synchrotron radiation facility SPring-8. A preliminary optical study is shown by Massahi et al. (in prep) where the absorption edges of single-layer iridium in the energy range from 50 to $800 \mathrm{eV}$ are presented. Here, two techniques were used: $\mathrm{x}$-ray reflectivity (XRR) and $\mathrm{x}$-ray photoelectron spectroscopy (XPS) to identify the absorption edges and emission lines of the material. It was shown that the surface of iridium film was contaminated with hydrocarbons displaying $\mathrm{C}$ and $\mathrm{O} \mathrm{K}$ edges. We also expect these contaminants on the iridium film investigated in this work. Section 2 provides the SPO mirror sample, facility of the x-ray beam line, and the method to measure the x-ray reflectance. Section 3 presents results of the reflectance measurements with angle scan and energy scan. The scattering factors $f_{1}$ and $f_{2}$ are estimated from our measurements in Sec. 4. In the estimation, we first employ a single-layer model to the measured reflectance and then employ an overlayer model as considered by Graessle et al. ${ }^{8}$ This paper presents our findings pertaining to the atomic scattering factors for iridium (and at all its L-edges) in the high-energy region.

\section{SPO Mirror Plate and X-Ray Measurement}

\subsection{SPO Mirror Plate}

SPO mirror plates are used to manufacture the SPO stacks, which are integrated in pairs in the mirror modules for the Athena optics. The mirror substrate is made from commercially available silicon wafers. ${ }^{13}$ The plate consists of two main functional parts: a membrane, which acts as the mirror, and ribs to interconnect multiple plates. To implement the Wolter-I type configuration of the stacked plates, an oxide wedge is grown along the ribbed direction of the SPO plate. The complete process to create the pore structure from silicon wafers has been described by Landgraf et al. ${ }^{14}$

The SPO plate considered in this study was diced to create a rectangular shape with a length and width of 110 and $49 \mathrm{~mm}$, respectively, corresponding to the inner radius modules of the Athena optics. For the purpose of coating characterization, the substrate surface has no

J. Astron. Telesc. Instrum. Syst. $\quad$ 014001-2 Jan-Mar 2021 • Vol. 7(1) 
photoresist patterning. The sample was prepared at the coating facility dedicated for the Athena mirror production. ${ }^{15}$ Specifically, the substrates were plasma cleaned prior to being coated, using the integrated inverse sputter etcher to remove any surface contamination and ensure a smooth surface. A 10-nm-thick iridium thin film coating was deposited using dc magnetron sputtering at a working pressure of $3.4 \times 10^{-3}$ mbar and magnetron discharge power of $1860 \mathrm{~W}$. A honeycomb collimation mesh was placed in the path between the iridium target and substrate to minimize the coating roughness.

\subsection{SPring-8}

We performed x-ray measurements of the SPO plate from January $18-22,2019$, at BL20B2 ${ }^{16}$ in SPring- 8 , which is a third-generation synchrotron radiation facility located in Hyogo, Japan. The acceleration energy of the electron beam was $8 \mathrm{GeV}$.

In general, BL20B2 is a medium-length beamline with a bending magnet as a light source, which is allocated for medical applications and developments of various imaging techniques in the energy range of 5 to $113 \mathrm{keV}$. The total length of this beamline is $215 \mathrm{~m}$ (from light source to end station). BL20B2 consists of an optics hutch and three experimental hutches 1, 2, and 3 located 42, 200, and $207 \mathrm{~m}$ from the light source, respectively. The x-ray beam, which is extracted from the bending magnet with a horizontal acceptance angle of $1.5 \mathrm{mrad}$, spreads to a 300-mmwide beam at hutch 2 ; therefore, the available maximum $\mathrm{x}$-ray beam size is $300 \mathrm{~mm}$ (horizontal) $\times$ $20 \mathrm{~mm}$ (vertical). The Si double crystal monochromator (DCM) ${ }^{17}$ which monochromatizes continuum $\mathrm{x}$-rays, is located in the optics hutch, $36.8 \mathrm{~m}$ from the light source. By changing the crystal planes of 111 (5.0 to $37.5 \mathrm{keV}), 311$ (8.4 to $72.5 \mathrm{keV}$ ), and 511 (13.5 to $113.3 \mathrm{keV}$ ), a wide energy range (5 to $113 \mathrm{keV}$ ) is available. In our experiment, we selected the crystal plane of 311 . The energy resolution $(\Delta E / E)$ of the available $\mathrm{x}$-ray beam is $<10^{-4}$ in this energy region.

Figure 1 shows the SPO plate placed in hutch 3. The $x$-rays arrived from the left side of Fig. 1. The direction of the $x$-ray beam and that perpendicular to the $x$-ray beam were defined as the $X$ and $Z$ axes, respectively. The sample chamber, which included the SPO plate, was mounted on the positioning stages, which allowed three-axis rotations $\left(\theta_{X}, \theta_{Y}\right.$, and $\left.\theta_{Z}\right)$ and two-axis translations $(Y$ and $Z$ ). The SPO plate was set in the sample chamber using an SPO holder made of Teflon. To monitor the intensity of the X-ray beam, an ionization chamber (IC1) was present in the front of the sample chamber. Moreover, another ionization chamber (IC2) was set behind the SPO sample to measure the intensity of the reflected x-rays.

The $\mathrm{x}$-ray beam was shaped to a size of $0.03 \mathrm{~mm}$ (vertical) $\times 0.5 \mathrm{~mm}$ (horizontal) by the fouraxis slits in the front of IC1. Since the divergence angle of the shaped $\mathrm{x}$-ray beam at $200 \mathrm{~m}$ from the light source was $<1$ arc sec, the beam size at IC2, which was located about $0.6 \mathrm{~m}$ behind the four-axis slit, is similar to the slit size.

Although air was used in IC1, argon was used instead of air to increase the detection efficiency in IC2. IC1 and IC2 had the same geometrical structure with horizontally long windows sized $8 \mathrm{~mm}$ (vertical) $\times 65 \mathrm{~mm}$ (horizontal). The reflected beam was moved into the vertical
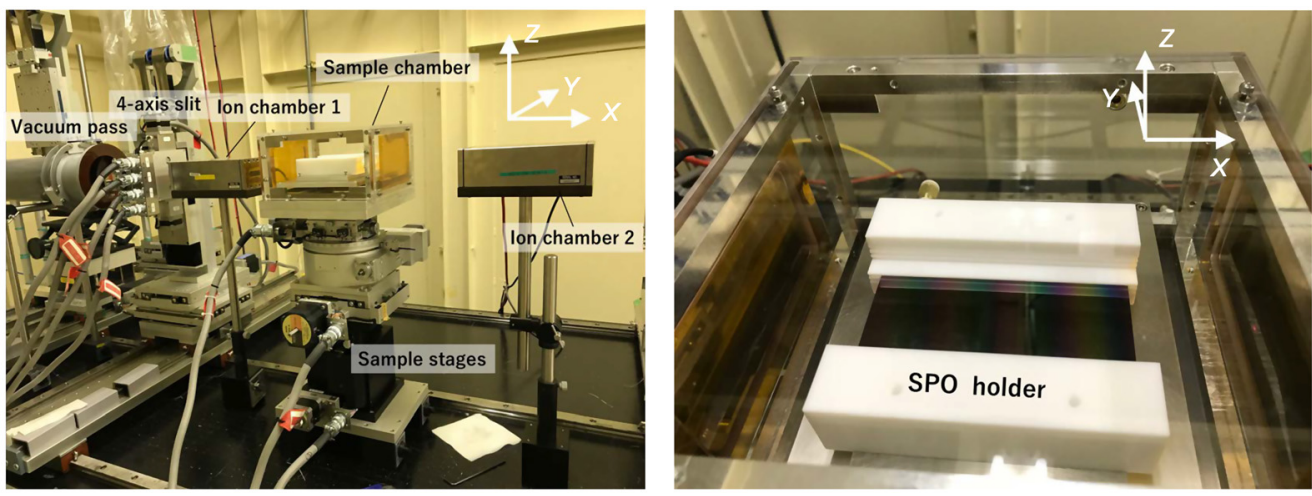

Fig. 1 Images of the experimental setup. The right image shows the inside of the sample chamber. 
direction $(Z)$ by changing the incident angle to the SPO sample. The vertical displacement of the beam at the entrance of IC2 was about $6.6 \mathrm{~mm}$ per degree of incident angle. IC2 was rotated $90 \mathrm{deg}$ around the $X$ axis so that the long direction of entrance window was vertical.

\subsection{X-Ray Measurements to Determine the Reflectance}

The reflectance $R$ is defined as the ratio of the intensity of a reflected x-ray to that of the incident $\mathrm{X}$-rays. Because the intensity of the incident X-rays may fluctuate, and IC1 and IC2 detect background events, we subtracted the background events and then corrected the fluctuation of the incident $\mathrm{x}$-rays. After incorporating the corrections, the reflectance can be defined as

$$
R=\frac{\left(I_{2 r}-I_{2 b}\right)\left(I_{1 d}-I_{1 b}\right)}{\left(I_{2 d}-I_{2 b}\right)\left(I_{1 r}-I_{1 b}\right)}
$$

where $I_{1}$ and $I_{2}$ denote the output counts of IC1 and IC2, respectively; and the subscripts $d, r$, and $b$ denote the incident x-rays, reflected x-rays, and background, respectively (see Fig. 2). In the case of our IC, the current from the IC was amplified with a transconductance (current to voltage) amplifier, and it was converted to pulses with a voltage to frequency $(V / F)$ converter and counted by a scaler. At the transconductance amplifier, we added an offset voltage to avoid a negative output. Thus, the outputs from IC1 and IC2 contain the offset at the transconductance amplifier, and the value obtained by subtracting the offset from the output of IC is proportional to the intensity of the incident $\mathrm{x}$-ray.

In general, the reflectance $R$ is described as a function of the energy $E$ of the incident x-ray and grazing angle $\theta$. Therefore, we measured the reflectivity by performing angle and energy scans at fixed energies and fixed incident angles, respectively.

The angle scans were performed to determine the surface roughness $(\sigma)$, thickness $(d)$ of the iridium coating, interfacial roughness $\left(\sigma_{b}\right)$ between the iridium layer and mirror substrate, and
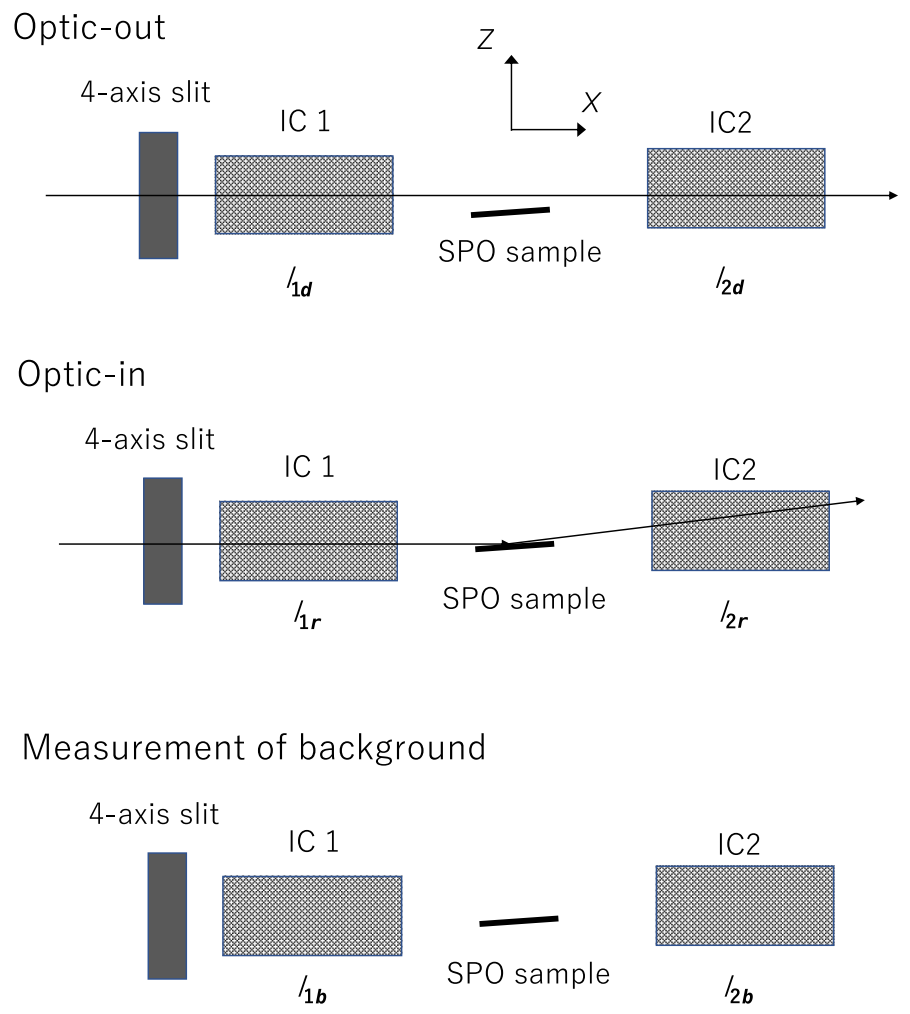

Fig. 2 Schematic of the configuration for each measurement. In the background measurement, the beam shutter upstream of the beam line was closed. The $\mathrm{x}$-ray beam was shaped with the fouraxis slit to a size of $0.03 \mathrm{~mm}$ (vertical) $\times 0.5 \mathrm{~mm}$ (horizontal). 
the atomic scattering factors $f_{1}$ and $f_{2}$ of iridium. Angle scans were performed at x-ray energies of 11,12 , and $14 \mathrm{keV}$, spanning the range of the iridium L-edges, ${ }^{6,18}$ specifically, the L3 edge $(11.2152 \pm 0.0003 \mathrm{keV}), \mathrm{L} 2$ edge $(12.8241 \pm 0.0003 \mathrm{keV})$, and L1 edge (13.4185 \pm $0.0003 \mathrm{keV})$. The incident angle was scanned up to $1.5 \mathrm{deg}$ in steps of $0.05 \mathrm{deg}$ measure the thickness of the iridium layer.

The energy scans were performed to measure the energy dependence of the atomic scattering factors with an exposure time per point of $3 \mathrm{~s}$. The wide energy region covering the iridium L-edges was scanned in two energy ranges: 9 to $13 \mathrm{keV}$ and 12 to $15 \mathrm{keV}$ in steps of $10 \mathrm{eV}$. This scan was referred to as a coarse pitch scan in this work. Furthermore, energy scans were performed around the energies of the iridium L-edges in steps of $1.5 \mathrm{eV}$ to clarify the iridium Lshell structure of $f_{1}$ and $f_{2}$. The energy step was smaller than the energy resolution of the X-IFU TES detector onboard Athena. This scan was referred as a fine pitch scan in this work. The incident angles in the energy scan were selected as $0.20 \mathrm{deg}, 0.32 \mathrm{deg}, 0.40 \mathrm{deg}, 0.50 \mathrm{deg}$, and $0.60 \mathrm{deg}$ to include the critical angles at 9 and $15 \mathrm{keV}$. However, owing to machine-time limitations, only the energy region of 9 to $13 \mathrm{keV}$ was covered at incident angles of $0.50 \mathrm{deg}$ and $0.60 \mathrm{deg}$.

\section{Results of the Reflectance Measurement}

\subsection{Characteristics of the Background of the Ionization Chamber}

The background outputs of IC1 and IC2, $I_{1 b}$ and $I_{2 b}$, which were measured before/after the angle and energy scans, were used to evaluate the stability of the background outputs of IC1 and IC2. Figure 3 shows the mean intensities of the backgrounds for each $3 \mathrm{~s}$ as a function of the elapsed time from 0:00 [Japan Standard Time (JST) $(=\mathrm{UTC}+9 \mathrm{~h}$ )] on January 20, 2019. The uncertainty is defined as the standard deviation of 5 or 20 background data points. $I_{1 b}$ and $I_{2 b}$ gradually decreased at $\Delta I / \Delta t=-0.708$ and -1.458 counts $/ 3 \mathrm{sh}^{-1}$, respectively. The time drift of the background intensities during each scan $(\Delta t<2 \mathrm{~h})$ was comparable with or less than the statistical uncertainty. Thus, the derived standard deviations were used as $I_{1 b}$ and $I_{2 b}$. Because no background measurements were performed before and after the energy scans at $\theta=0.32 \mathrm{deg}$ and $0.40 \mathrm{deg}$, the average background outputs of IC1 and IC2, which were estimated to be 268.8 and 480.5, respectively, were applied for $I_{1 b}$ and $I_{2 b}$ in the energy scans.

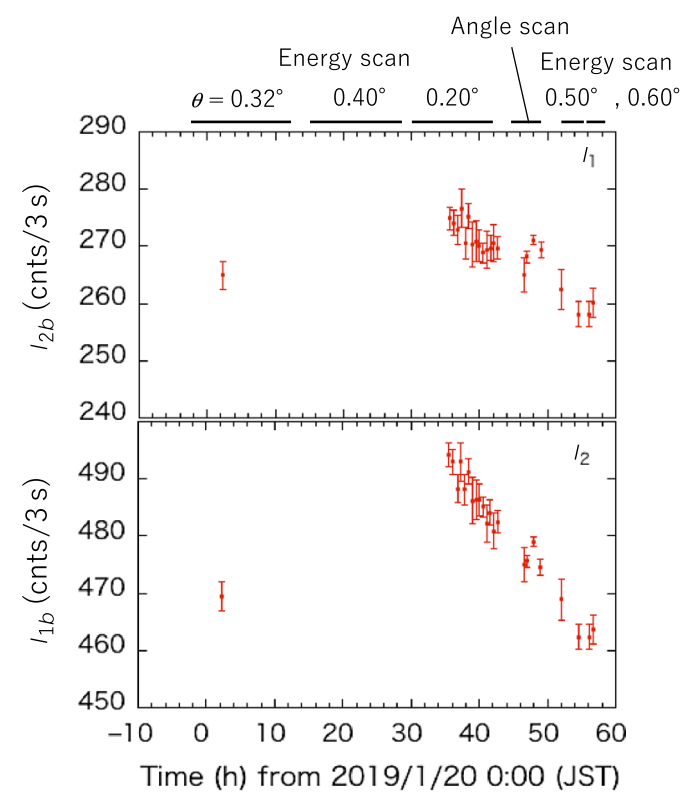

Fig. 3 Variability in the background intensities $I_{1 b}$ and $I_{2 b}$. The uncertainties in these intensities are defined as the standard deviations of 5 or 20 measurements. A log of the scans in the experiment is presented above the figure. 


\subsection{Energy Calibration}

To calibrate the energy scale, a thin $\mathrm{Cu}$ foil was placed on the window on the downstream side of IC1. Subsequently, we measured the output counts of IC1 and IC2 in the energy range from 8.9 to $9.1 \mathrm{keV}$ with a step of $0.5 \mathrm{eV}$. The exposure time was $1 \mathrm{~s}$, and the vertical slit size was $30 \mu \mathrm{m}$. The energy calibration was performed four times during the experiment. Figure 4 shows $-\log \left(I_{2} / I_{1}\right)$ as a function of the energy; the value of $-\log \left(I_{2} / I_{1}\right)$ corresponds to $\mu t$, where $\mu$ and $t$ denote the absorption coefficient and $\mathrm{Cu}$ foil thickness, respectively. The absorption edge energy was defined as the energy with the maximum derivative of $\mu t$. In the fitting with a Gaussian model, the peak value was $8977.3 \mathrm{eV}$ (right panel in Fig. 4). All of the energy calibration data points were treated in the same manner, and all the peak energies were noted to be consistent within $0.1 \mathrm{eV}$. Table 1 presents the results of the energy calibrations. The peak energy is identified as that of the $\mathrm{Cu} \mathrm{K}$ pre-edge $(8980.4 \mathrm{eV}) .{ }^{19}$ Thus the peak energy was $-3.1 \mathrm{eV}$ less than the energy of the pre-edge. This offset was corrected to assume a fixed angular offset of $0.009 \mathrm{deg}$ in the angle of the DCM.

\subsection{Calibration of the Incident Angle}

The incident angle to the SPO plate was changed by operating the swivel stage in the sample stages, and it was calibrated by obtained an x-ray image of the reflected light using an sCMOSbased visible light conversion type $\mathrm{x}$-ray image detector (sCMOS). The sCMOS was placed $2362 \mathrm{~mm}$ from the center of the SPO plate. A narrow beam with dimensions of $0.03 \mathrm{~mm}(Z)$ and $5 \mathrm{~mm}(Y)$, respectively, was reflected on the SPO plate at a swivel of $\theta_{Y}$. Using the sCMOS, we measured the beam displacement between the reflection and translating the optic out of the beam. The incident angle $\theta_{I}$ was calculated from the displacement and the relation between $\theta_{I}$ and $\theta_{Y}$ was determined. In the angle scan, we estimated $\theta_{I}$ using this relation, whereas in the energy scan, $\theta_{I}$ was fixed using the sCMOS.
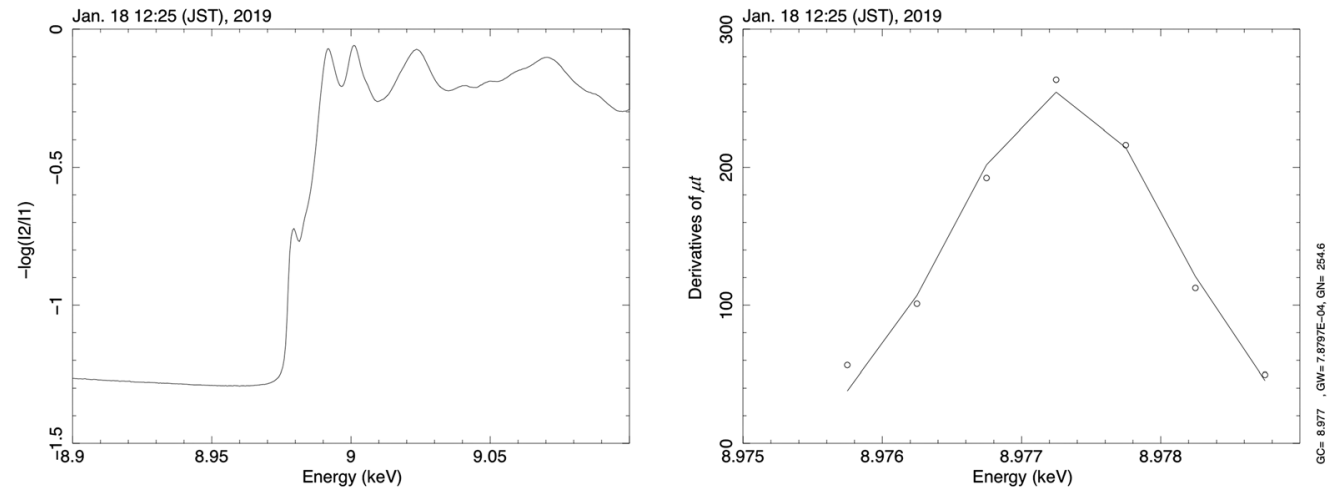

Fig. 4 Transmissivity of the $\mathrm{Cu}$ thin foil. $-\log (/ 2 / / 1)$ in the left panel corresponds to $\mu t$, where $\mu$ and $t$ denote the absorption coefficient and absorber thickness, respectively. The maximum derivatives of $\mu t$ were obtained by fitting with a Gaussian model.

Table 1 Energy calibration results.

\begin{tabular}{lc}
\hline \hline Time and date (JST) & Peak energy (eV) \\
\hline 12:25; January 18, 2019 & 8977.3 \\
21:00; January 19, 2019 & 8977.3 \\
13:05; January 20, 2019 & 8977.4 \\
04:45; January 21, 2019 & 8977.3 \\
\hline \hline
\end{tabular}



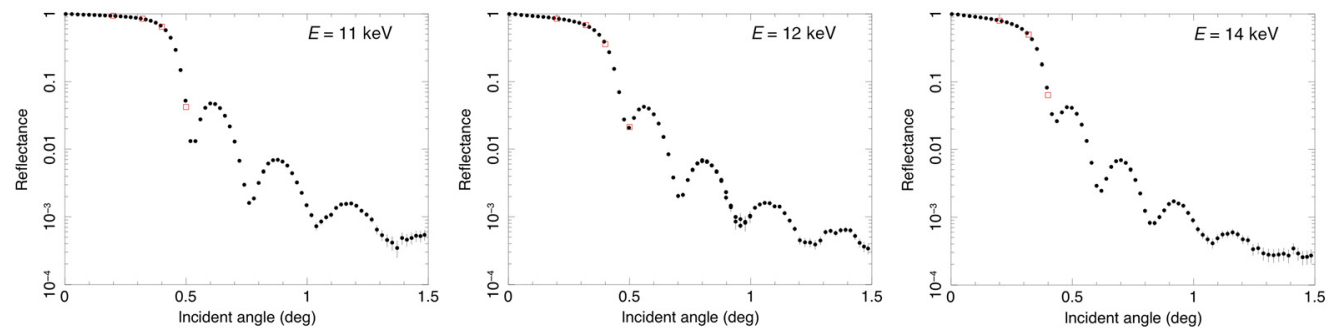

Fig. 5 X-ray reflectance obtained using the angle scan. The $x$-ray reflectance obtained using the energy scan is plotted as red open squares.

\subsection{Reflectance Obtained from the Angle Scan}

Before starting the angle scan, the outputs in optic-out $\left(I_{1 d}\right.$ and $\left.I_{2 d}\right)$ and backgrounds $\left(I_{1 b}\right.$ and $I_{2 b}$ ) were obtained five times. The mean values of the outputs were calculated for estimating the reflectance using Eq. (1). Figure 5 shows the reflectance as a function of $\theta_{I}$. The interference fringes resulting from a thin iridium layer with a thickness of $\sim 10 \mathrm{~nm}$ were observed at large incidence angles. To verify the consistency between the angle and energy scans, the results obtained using the energy scan were plotted (red open squares in Fig. 5). A reasonable agreement between the two scans can be observed.

Next, we estimated the error on the reflectance. The outputs, $I_{1 r}$ and $I_{2 r}$ in optic-in could not follow Poisson statistics, because we added the offset at the transconductance amplifier. Since the output of IC2 in optic-in consists of two components, outputs of IC2 in beam off and outputs originated from the reflected light. The error on the former component was estimated as the standard deviation on the background output, and the error on the latter component could be expressed by the statistical error on the measured x-ray intensity. Specifically, the errors $\Delta I_{1 r}$ and $\Delta I_{2 r}$ could be represented as

$$
\left.\begin{array}{l}
\Delta I_{1 r}^{2}=\Delta I_{1 b}^{2}+a_{1}^{2}\left(I_{1 r}-I_{1 b}\right) \\
\Delta I_{2 r}^{2}=\Delta I_{2 b}^{2}+a_{2}^{2}\left(I_{2 r}-I_{2 b}\right)
\end{array}\right\},
$$

where $a_{1}$ and $a_{2}$ are the factors representing the statistical error on the x-ray intensity. The factors were estimated from the outputs in optic-out. For instance, $\Delta I_{1 d}^{2}=\Delta I_{1 b}^{2}+a_{1}^{2}\left(I_{1 d}-I_{1 b}\right)$, where $\Delta I_{1 d}$ and $\Delta I_{1 b}$ are the standard deviations of the five measurements before starting the angle scan. The factors $a_{1}^{2}$ and $a_{2}^{2}$ depend on the x-ray energy. $a_{1}^{2}$ at $E=11,12$, and $14 \mathrm{keV}$ was calculated as $0.0098,0.0116$, and 0.1269 , respectively, and $a_{2}^{2}$ at $E=11,12$, and $14 \mathrm{keV}$ was calculated as $0.036,0.146$, and 2.517 , respectively. The error on the reflectance was estimated using the error of $I_{1 r}$ and $I_{2 r}$ represented in Eq. (2).

\subsection{Reflectance Obtained by Performing the Energy Scan}

After recording $I_{1 b}$ and $I_{2 b} 20$ times, we measured the intensities of the incident x-ray beam, $I_{1 d}$ and $I_{2 d}$, and those of the reflected x-rays, $I_{1 r}$ and $I_{2 r}$. The exposure time was $3 \mathrm{~s}$. The measurements were conducted two times to evaluate the reproducibility. The measurements were obtained in the following order: optic-out, optic-in, optic-in, and optic-out. However, the first energy scan at $\theta_{I}=0.6 \mathrm{deg}$ failed owing to issues with the data acquisition. Thus, only the second measurement was considered. The parameters for the energy scan are listed in Table 2. Figure 6 shows the mean reflectance of two measurements obtained in the energy scan. The iridium L-edge structure of the reflectance is shown in Fig. 7. In the case of the energy scans at $\theta_{I}=0.32 \mathrm{deg}$ and $0.40 \mathrm{deg}$, the average backgrounds were adopted as $I_{1 b}$ and $I_{2 b}$ to derive the reflectance.

The errors on the reflectance at energy $E_{i}$ were assumed to be reproducibility, which is the variation between the two measurements, and were estimated from the relative deviation $\delta_{i}^{j}$ of each measurement $R_{i}^{j}(j=1$ or 2$)$ from the mean value $m_{i}$. The relative deviation was defined as 
Table 2 Parameters for the energy scan.

\begin{tabular}{ll}
\hline \hline Incident angle $\theta_{l}(\mathrm{deg})$ & \multicolumn{1}{c}{ Scanning energy range (step) (eV) } \\
\hline 0.20 & 9000 to $13,000(10), 12,000$ to $15,000(10)$ \\
& 11,000 to $11,500(1.5), 12,500$ to $13,000(1.5), 13,200$ to $13,700(1.5)$ \\
& 9000 to $13,000(10), 12,000$ to $15,000(10)$ \\
0.32 & 11,000 to $11,500(1.5), 12,500$ to $13,000(1.5), 13,200$ to $13,700(1.5)$ \\
0.40 & 9000 to $13,000(10), 12,000$ to $15,000(10)$ \\
& 11,000 to $11,500(1.5), 12,500$ to $13,000(1.5), 13,200$ to $13,700(1.5)$ \\
0.50 & 9000 to $13,000(10)$ \\
0.60 & 9000 to $13,000(10)$ \\
\hline \hline
\end{tabular}
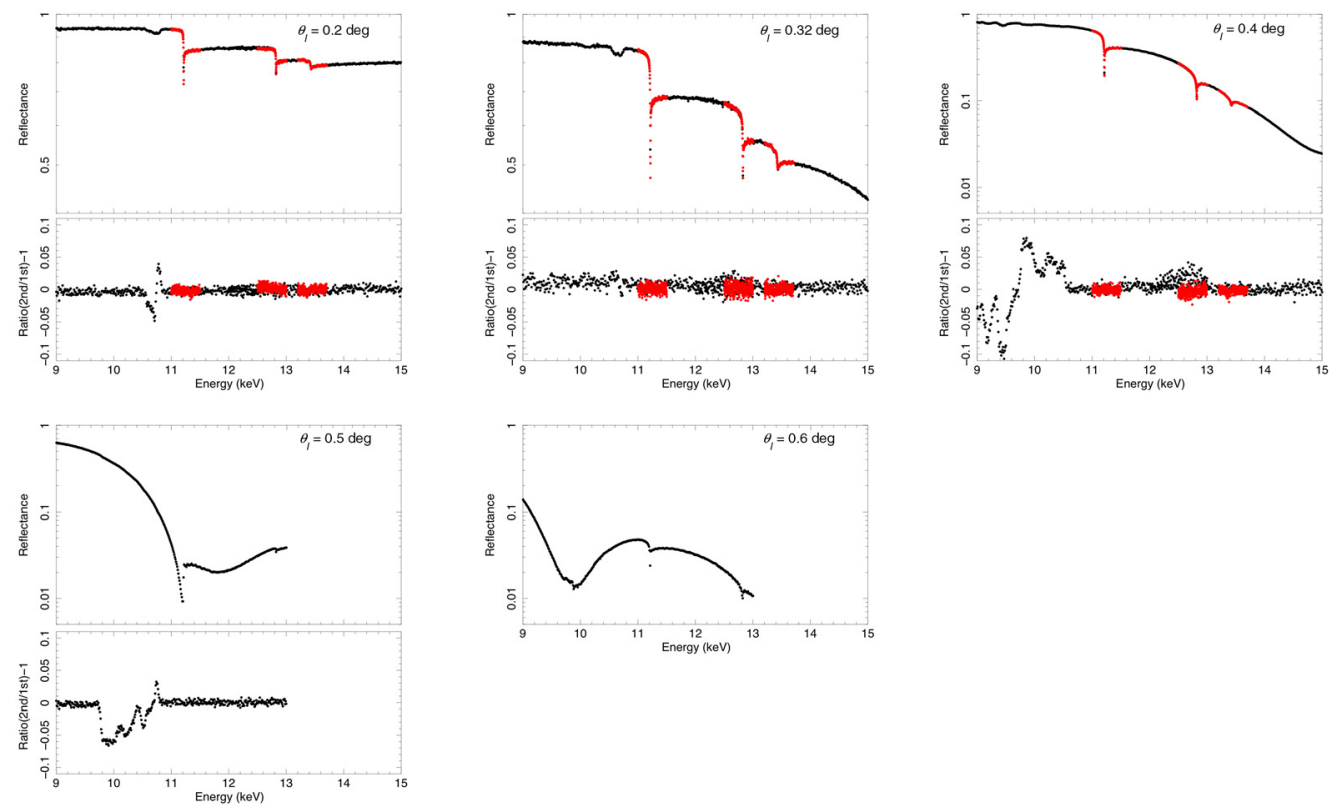

Fig. 6 Mean reflectance obtained in the energy scan with coarse and fine pitches. The black and red data present the results obtained from the coarse and fine pitch scans, respectively. The ratios between the two measurements are also plotted. In the energy scan at $\theta_{l}=0.6 \mathrm{deg}$, the first measurement failed, thus the corresponding ratio is not plotted.

$\delta_{i}^{j}=\left(R_{i}^{j}-m_{i}\right) / m_{i}$, where $i$ is the index of the scan data. The root mean square (rms) of $\delta_{i}$ was considered as the relative error in the reflectance and defined as follows:

$$
\mathrm{RMS}=\sqrt{\frac{1}{N} \sum\left\{\left(\delta_{i}^{1}\right)^{2}+\left(\delta_{i}^{2}\right)^{2}\right\}}
$$

where $N$ is the total number of data points. By considering the ratio $r_{i}\left(=R_{i}^{1} / R_{i}^{2}\right)$ of the two measurements of reflectance and considering that $r_{i}+1$ to 2 , the following expression can be obtained

$$
\left(\delta_{i}^{1}\right)^{2}+\left(\delta_{i}^{2}\right)^{2}=2\left(\frac{r_{i}-1}{r_{i}+1}\right)^{2} \sim \frac{1}{2}\left(r_{i}-1\right)^{2} .
$$



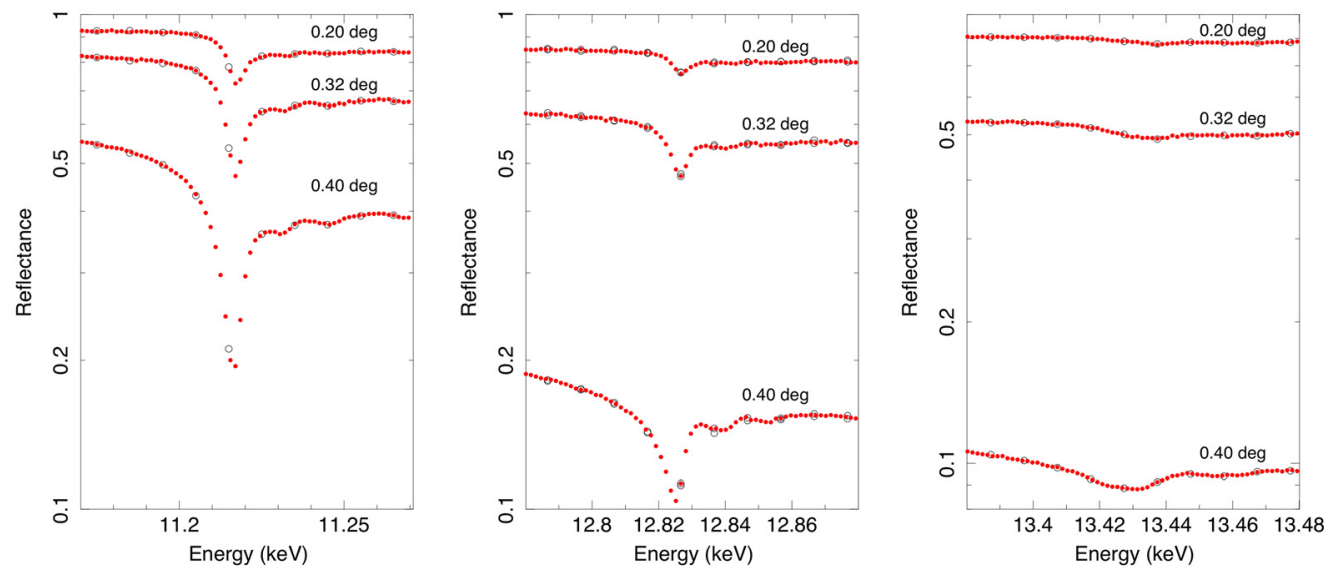

Fig. 7 Iridium L-edge structure of the reflectance obtained by the energy scan. The black circles and red data present the results obtained from the coarse and fine pitch scans, respectively.

Then

$$
\mathrm{RMS}=\sqrt{\frac{1}{N} \sum \frac{1}{2}\left(r_{i}-1\right)^{2}} .
$$

The bottom panels in Fig. 6 display the values of $r_{i}-1$. The data were grouped in $500 \mathrm{eV}$ batches. It was assumed that the relative errors within a data group were the same, and the error was estimated using Eq. (5). The relative errors in the energy region above $11 \mathrm{keV}$ were estimated to be $\sim 0.3 \%$ to $0.5 \%$. The angle scan data at $E=12 \mathrm{keV}$ were obtained with an exposure time of $3 \mathrm{~s}$, and the relative error was estimated to be $\sim 0.46 \%$ at incident angles below $0.4 \mathrm{deg}$. This value is similar to the relative error estimated using Eq. (2).

In the energy region below $11 \mathrm{keV}$, the ratio deviated from unity. The discrepancy led to the reflectance exhibiting a large error of about $5 \%$ at the specific energies Although the underlying cause was not further examined, the large discrepancy may be caused by the contamination with unexpected higher-order x-ray photons to the incident x-rays. In the case of $\theta_{I}=0.32 \mathrm{deg}$, a small dip at $\sim 10.6 \mathrm{keV}$ was reproduced in the two measurements. Owing to the reproducibility of the dip structure, the dip structure had small error and affected the calculation of the atomic scattering factors (see Fig. 10).

\section{Estimating the Atomic Scattering Factors}

\subsection{Reflectance of a Single Layer}

The reflectance from any interface can be calculated using Fresnel's equations by employing the continuity conditions for incident, reflected, and transmitted waves. Figure 8 schematically illustrates a mirror substrate coated with a thin layer with a thickness $d_{1}$. The refractive indices of the substrate, thin layer, and medium (air) above the layer are defined as $\tilde{n}_{0}, \tilde{n}_{1}$, and $\tilde{n}_{2}$, respectively. The refractive index of an x-ray with an energy $E$ is expressed as

$$
\tilde{n}(E)=1-\delta(E)+i \beta(E)
$$

where $\delta(E)$ and $\beta(E)$ denote the dispersion and absorption terms, respectively, and are related to the atomic scattering factors $f_{1}$ and $f_{2}$ as

$$
\delta=\frac{r_{e} N_{0} \rho}{2 \pi A} \lambda_{0}^{2} f_{1}, \quad \beta=\frac{r_{e} N_{0} \rho}{2 \pi A} \lambda_{0}^{2} f_{2},
$$

where $N_{0}$ is the Avogadro constant, $r_{e}$ is the classical electron radius, $A$ is the atomic weight, $\rho$ is the mass density, and $\lambda_{0}$ is the $\mathrm{x}$-ray wavelength. 


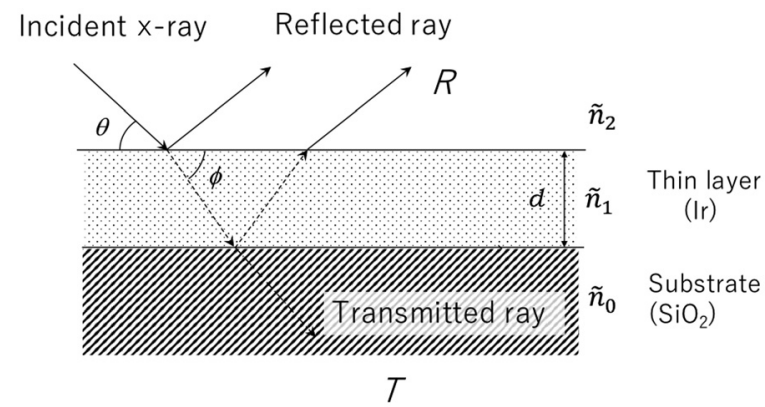

Fig. 8 Reflection on a single thin layer. $R$ and $T$ denote the reflectivity and transmission coefficients, respectively.

The reflectivity coefficient $R$ can be expressed as

$$
R=\frac{r_{1}+r_{0} \exp \left(-i \Delta_{1}\right)}{1+r_{0} r_{1} \exp \left(-i \Delta_{1}\right)}
$$

where $r_{1}$ and $r_{0}$ denote the Fresnel reflectivity coefficients of the two interfaces (air/thin layer and thin layer/substrate, respectively). $\Delta_{1}$ corresponds to a phase shift; $\Delta_{1}=\left(4 \pi / \lambda_{0}\right) \tilde{n}_{1} d_{1} \sin \phi$. The reflectivity of the thin layer is deduced as a square of $R$. If the surfaces of the thin layer and substrate interface are not smooth, the loss of reflectance owing to roughness must be considered. In this work, the Nevot-Croce factor ${ }^{20}$ was considered to take into account the effect of roughness. The reflectance and effect of the roughness were determined using the subroutines multilayerRefl and calcRoughness, of xrtreftable in heasoft $6.25,{ }^{21}$ respectively.

To determine the optimal parameters that could reproduce the measurements, the MINUIT package developed by CERN to determine the minimum value of a multiparameter function and analyze the shape of the function around the minimum, was used. This software package was used to determine the optimal fit parameters with the minimum value of $\chi^{2}$.

\subsection{Analysis of Angle Scan Data}

The fit to the angle scan data was used to identify the parameters describing the iridium layer, specifically, the thickness of the iridium layer $(d)$, interfacial roughness between the iridium layer and SPO substrate $\left(\sigma_{b}\right)$, and the surface roughness of the iridium layer $(\sigma)$. The atomic weights of iridium and the substrate $\left(\mathrm{SiO}_{2}\right)$ were 192.22 and 60.083 , respectively, and the corresponding mass densities were 22.421 and $2.65 \mathrm{~g} \mathrm{~cm}^{-3}$, respectively.

First, we fitted the angle scan data in the angular region between 0.1 deg and $1.5 \mathrm{deg}$ using model 1 in which $\sigma=\sigma_{b}$. In the fitting procedure, $f_{1}$ and $f_{2}$ of the substrate were set constant as those of $\mathrm{SiO}_{2}$. Table 3 lists the best fit parameters, and the best fit model is overlaid on the data with a dashed line in Fig. 9. Although the best fit values of $f_{1}, f_{2}, d$, and $\sigma$ were as expected, model 1 yielded a large $\chi^{2}$ value due to a large discrepancy at the minimum of the interference pattern. Subsequently, model 2 , in which the interfacial roughness $\left(\sigma_{b}\right)$ was considered to be variable, was used to reproduce the interference pattern. Although the values of $\chi^{2}$ reduced to $\sim 1 / 4$, the values were still large due to the discrepancy at $\theta_{I}>1$ deg (Fig. 9).

Although the measurements for $\theta_{I}>1 \mathrm{deg}$ were effective for reproducing the interference pattern, it was efficient to use the data at the angle near the critical angle to determine $f_{1}$ and $f_{2}$. The data at $\theta_{I}<0.6 \mathrm{deg}$ were also less affected by background uncertainty. Thus, we fitted the angle scan data at the angular region $\theta_{I}<0.6$ deg using model 2 . The best fit iridium thickness involved a large uncertainty because only one interference pattern was present at $\theta_{I}<0.6 \mathrm{deg}$. Thus, we fixed the thickness $d$ as $10.04 \mathrm{~nm}$, which was the mean value of the best fit thickness in model 2. The data were well reproduced with the $\chi^{2}$ reduced by $\sim 1$ (Fig. 10). Table 5 lists the best fit parameters in model 2. Comparing the best fit values listed in Tables 4 and 5 , it was noted that $f_{1}$ and $f_{2}$ are consistent within $1 \%$. When the data were fitted in the angular region $\theta_{I}<1.0 \mathrm{deg}$, the best fit $f_{1}$ and $f_{2}$ were consistent with the best fit results at $\theta_{I}<0.6 \mathrm{deg}$, 
Awaki et al.: Measuring the atomic scattering factors near the iridium L-edges...

Table 3 Best fit parameters when using model 1.

\begin{tabular}{lccc}
\hline \hline & $11 \mathrm{keV}$ & $12 \mathrm{keV}$ & $14 \mathrm{keV}$ \\
\hline Ir $f_{1}$ & $66.15(66.5)$ & $68.49(69.54)$ & $71.42(72.4)$ \\
Ir $f_{2}$ & $4.29(4.13)$ & $9.43(9.07)$ & $11.52(11.2)$ \\
Thickness $d(\mathrm{~nm})$ & 10.10 & 10.08 & 10.06 \\
Surface roughness $\sigma\left(=\sigma_{b}\right)(\mathrm{nm})$ & 0.45 & 0.41 & 0.37 \\
$\mathrm{SiO}_{2} f_{1}$ (constant) & 10.08 & 10.07 & 10.05 \\
$\mathrm{SiO}_{2} f_{2}$ (constant) & 0.0701 & 0.0589 & 0.0433 \\
$\chi^{2} /$ degree of freedom (d.o.f.) & $2557.4 / 65$ & $1853.1 / 88$ & $784.1 / 65$ \\
\hline \hline
\end{tabular}

$f_{1}$ and $f_{2}$ reported by Henke et al. ${ }^{6}$ are presented in ( ).
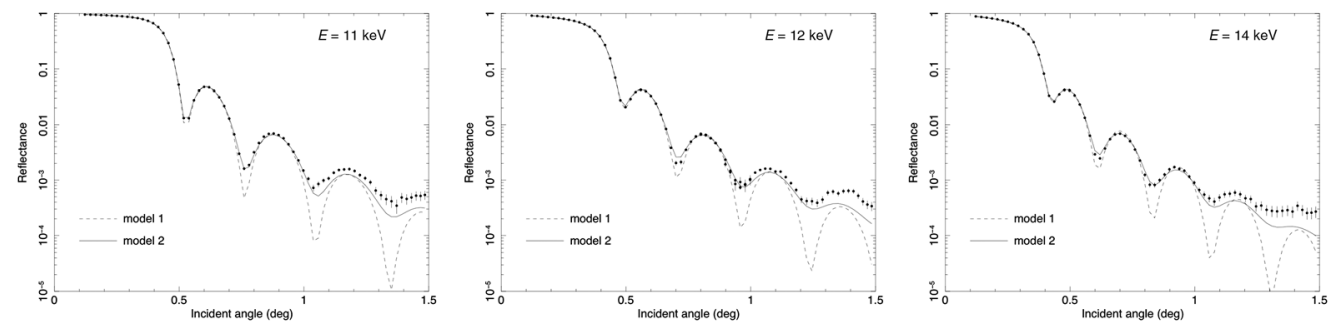

Fig. 9 Angle scan data obtained using the best-fit curves of models 1 and 2 (dashed and solid lines, respectively).
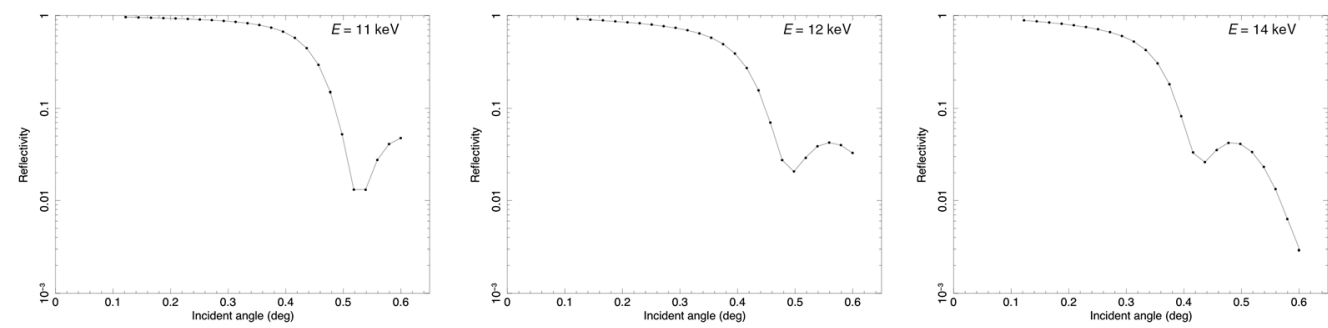

Fig. 10 Angle scan data at $\theta_{l}<0.6 \mathrm{deg}$. The solid line in each panel displays the best fit model (model 2).

Table 4 Best fit parameters when using model 2.

\begin{tabular}{lccc}
\hline \hline & $11 \mathrm{keV}$ & $12 \mathrm{keV}$ & $14 \mathrm{keV}$ \\
\hline Ir $f_{1}$ & 65.57 & 67.55 & 70.42 \\
$\operatorname{Ir} f_{2}$ & 4.01 & 8.76 & 10.87 \\
Thickness $d(\mathrm{~nm})$ & 10.05 & 10.05 & 10.01 \\
Surface roughness $\sigma(\mathrm{nm})$ & 0.27 & 0.23 & 0.22 \\
Interfacial roughness $\sigma_{b}(\mathrm{~nm})$ & 0.657 & 0.676 & 0.651 \\
$\chi^{2} /$ d.o.f. & $358.6 / 64$ & $462.6 / 87$ & $191.7 / 64$ \\
\hline \hline
\end{tabular}


Table 5 Best fit parameters obtained by fitting the angle scan data at $\theta_{l}<0.6$ deg using model 2 .

\begin{tabular}{lccc}
\hline \hline & $11 \mathrm{keV}$ & $12 \mathrm{keV}$ & $14 \mathrm{keV}$ \\
\hline $\operatorname{Ir} f_{1}$ & $65.59 \pm 0.02$ & $67.85 \pm 0.05$ & $70.88 \pm 0.08$ \\
$\operatorname{Ir} f_{2}$ & $4.02 \pm 0.01$ & $8.87 \pm 0.05$ & $11.02 \pm 0.07$ \\
Thickness $d(\mathrm{~nm})$ (constant) & 10.04 & 10.04 & 10.04 \\
Surface roughness $\sigma(\mathrm{nm})$ & $0.296 \pm 0.005$ & $0.319 \pm 0.009$ & $0.307 \pm 0.011$ \\
Interfacial roughness $\sigma_{b}(\mathrm{~nm})$ & $0.641 \pm 0.005$ & $0.650 \pm 0.012$ & $0.654 \pm 0.016$ \\
$\chi^{2} /$ d.o.f. & $16.7 / 20$ & $12.4 / 20$ & $11.2 / 20$ \\
\hline \hline
\end{tabular}

Error ranges represent $1 \sigma$ confidence boundaries for a parameter of interest $\left(\chi_{\min }^{2}+1\right)$.

within $0.7 \%$. This indicates that fitting to the angle scan data at $\theta_{I}<0.6$ deg can produce nearly the same results as fitting the data up to 1 deg or $1.5 \mathrm{deg}$.

The uncertainty presented in Table 5 represents the $1 \sigma$ confidence boundary for a parameter of interest $\left(\chi_{\min }^{2}+1\right)$, where $\chi_{\min }^{2}$ is the minimum value of $\chi^{2} . f_{1}$ and $f_{2}$ were $\sim 98 \%$ of the values reported by Henke et al. ${ }^{6}$ Moreover, the coating surface of the SPO plate was noted to be smooth, with a roughness of $\sim 0.3 \mathrm{~nm}$. This result indicates that iridium was successfully deposited on the silicon substrate. The surface roughness of iridium layers deposited on SPOs has been investigated using AFM measurements in Girou et al. ${ }^{22}$ It is observed that the surface roughness of iridium layers is between 0.2 and $0.3 \mathrm{~nm}$ similar to the roughness presented in this work. We observed a lower roughness of the iridium surface roughness compared to the interface roughness, which indicates that the amorphous iridium layers smooth out the substrate surface roughness. This is also observed in Chandra.

\subsection{Analysis of Energy Scan Data}

The energy scan data involved a few angle data points for each energy, and the measured values were fitted in terms of $f_{1}$ and $f_{2}$ of the iridium coating layer. In the fitting procedure, $\sigma$ and $\sigma_{b}$ were set constant as the mean values of the best-fit values obtained from the angle scan, that is, 0.307 and $0.648 \mathrm{~nm}$, respectively. As the error in the scan data at $\theta_{I}=0.6$ deg was not estimated, these data were used not for fitting but for verifying the fitting results. Most of the energy scan data were well fitted with a reduced $\chi^{2}<2.5$, although one data set at $E=11213.1 \mathrm{eV}$ had a large $\chi^{2}$ of $\sim 5$.

Figure 11 shows the best fit $f_{1}$ and $f_{2}$ derived from the SPO plates. All the data obtained from the coarse (black dots) and fine pitch (red dots) scans were plotted. $f_{1}$ and $f_{2}$ at 11,12 , and $14 \mathrm{keV}$, derived from the angle scan data, were overlaid in Fig. 11 with open circles. The scattering factors derived from the angle scan were consistent with those obtained from the energy scan within $1 \%$. The two peaks of $f_{2}$ near $10.7 \mathrm{keV}$ were attributed to the valley of the reflectance at $0.32 \mathrm{deg}$, as observed in Fig. 6. Ignoring the energy scan data at $0.32 \mathrm{deg}$ in the energy region of 10.4 to $10.8 \mathrm{keV}$, we recalculated $f_{1}$ and $f_{2}$, and there were no clear peaks near $10.7 \mathrm{keV}$ (Fig. 13).

Furthermore, the amount of data obtained from the fine pitch scan around the iridium L3 edge was slightly less than that obtained from the coarse pitch scan between 9 and $13 \mathrm{keV}$. A possible reason is that in the coarse pitch scan, we used four data points at $0.2 \mathrm{deg}, 0.32 \mathrm{deg}, 0.4 \mathrm{deg}$, and $0.5 \mathrm{deg}$ to determine $f_{1}$ and $f_{2}$, whereas only three angles, $0.2 \mathrm{deg}, 0.32 \mathrm{deg}$, and $0.4 \mathrm{deg}$, were considered in the fine pitch scan. To find out the effect of the difference of the number of data points, we derived $f_{1}$ and $f_{2}$ without using the coarse pitch data at $0.5 \mathrm{deg}$, and the obtained results were noted to be similar to those of the fine pitch scan. Figure 12 shows the ratio of the results with and without the data obtained at $0.5 \mathrm{deg}$. At an energy of more than $\sim 12 \mathrm{keV}$, the ratio was nearly unity; however, at energies less than $\sim 12 \mathrm{keV}$, the ratios for $f_{1}$ and $f_{2}$ were $1.2 \%$ and $4.5 \%$ less than unity, respectively. As the critical angle for iridium at $12 \mathrm{keV}$ is $\sim 0.385 \mathrm{deg}$, the above finding likely indicates that a systematic uncertainty occurs unless the 


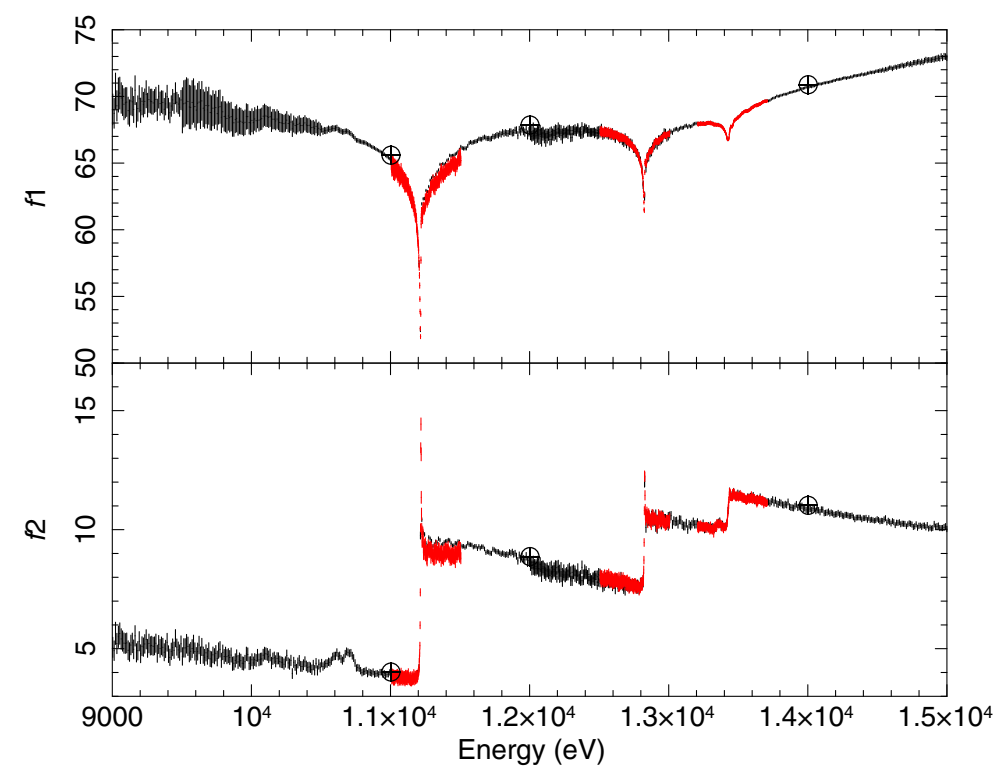

Fig. 11 Atomic scattering factors obtained in the energy scan. The black and red data present the results obtained from the coarse and fine pitch scans, respectively. The results of the angle scan at $E=11,000,12,000$, and $14,000 \mathrm{eV}$ are also plotted as open circles.

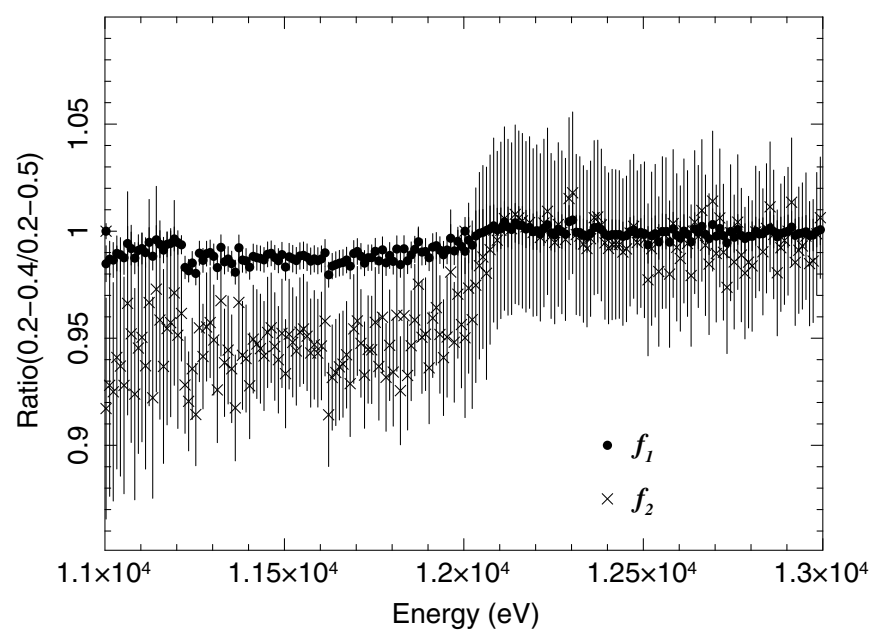

Fig. 12 Ratio of atomic scattering factors obtained when the data obtained at 0.5 deg are included to those when they are not included.

data obtained at an angle greater than the critical angle are used. When the data for the coarse pitch scan at 9 to $13 \mathrm{keV}$ were fit with the data obtained at $0.6 \mathrm{deg}$, the results were in agreement with those of $0.5 \mathrm{deg}$ within $1 \%$.

Figure 13 shows the corrected $f_{1}$ and $f_{2}$ obtained by adopting the ratio shown in Fig. 12. Specifically, in the energy region of 12 to $13 \mathrm{keV}$, the atomic scattering factors were derived from the coarse pitch scans of both 9 to $13 \mathrm{keV}$ and 12 to $15 \mathrm{keV}$. The average values of the two measurements are plotted in Fig. 13. The corrected $f_{1}$ and $f_{2}$ of the fine pitch scan around L3 edge are consistent with those obtained from the coarse pitch scan between 9 and $13 \mathrm{keV}$.

Figure 14 shows the derived $f_{1}$ and $f_{2}$ near the iridium L-edges to clarify the iridium L-edge structures. The energies of the iridium L-edges ${ }^{6,18}$ are indicated with dashed lines. The peak and oscillation structures of $f_{2}$ near the iridium L3 and L2 edges are known as the x-ray absorption near edge structure (XANES) ${ }^{23}$ and the peaks indicate the absorption threshold resonances associated with the excitation of $2 p$ electrons to unfilled $d$ states in the valence band. ${ }^{23,24}$ Because the 


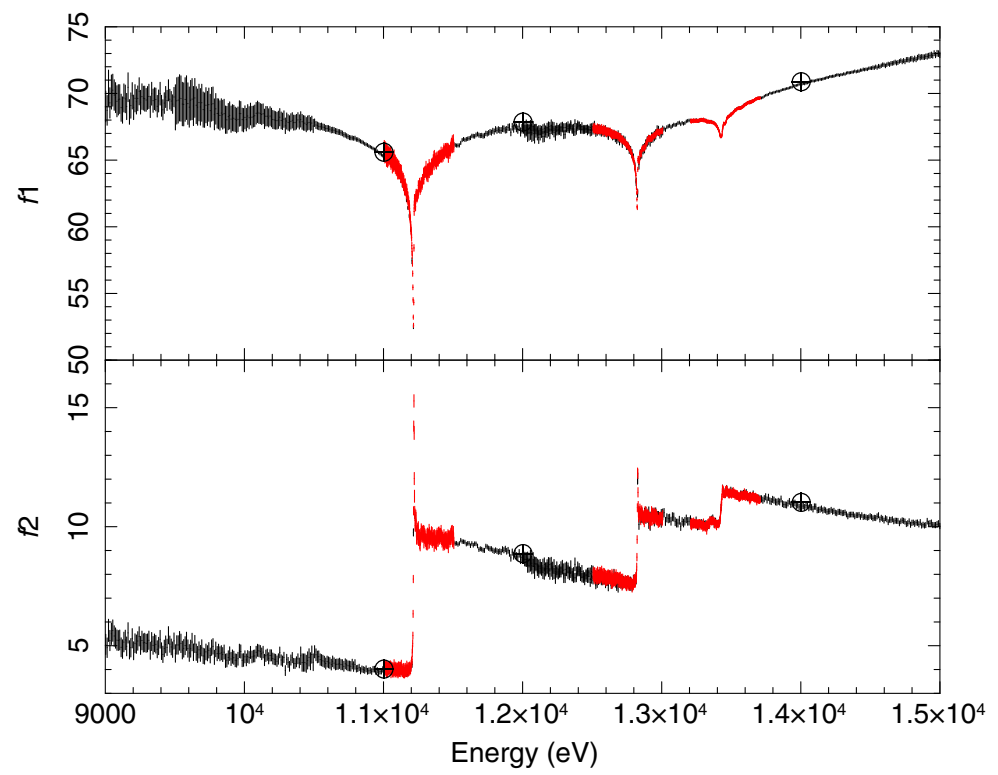

Fig. 13 The atomic scattering factors $f_{1}$ and $f_{2}$ of iridium corrected with the ratios shown in Fig. 12 . The results obtained using the fine pitch scan are plotted in red. The results from the angle scans at $11,000,12,000$, and $14,000 \mathrm{eV}$ are indicated by open circles.

L1 edge corresponds to the excitation of the electrons from the $2 s$ states, no peak occurs near the L1 edge. To characterize the L-edge structures, we fitted the $f_{2}$ obtained from the fine pitch scans with a Lorentzian + arctan model in which a Lorentzian represents a sharp line and an arctan represents a step-like edge feature. ${ }^{25}$ The L-edge structures were well represented by this model as seen in Fig. 14. The best values are listed in Table 6. The best fit parameters of the L3 edge were consistent with those by Monteseguro et al. ${ }^{25}$ The center energy of the arctan model representing a step-like edge feature is consistent with the L3 and L2 edge energies, although the best fitted center energy for iridium L1 edge is $\sim 8 \mathrm{eV}$ greater than the iridium L1 edge energy. Although the recommended value of the L1 edge energy was $13418.5 \mathrm{eV},{ }^{18}$ Bearden $(1967)^{26}$ reported an L1 edge energy of 13,423 eV from his measurement. Our result was close to the measurement of Bearden (1967).
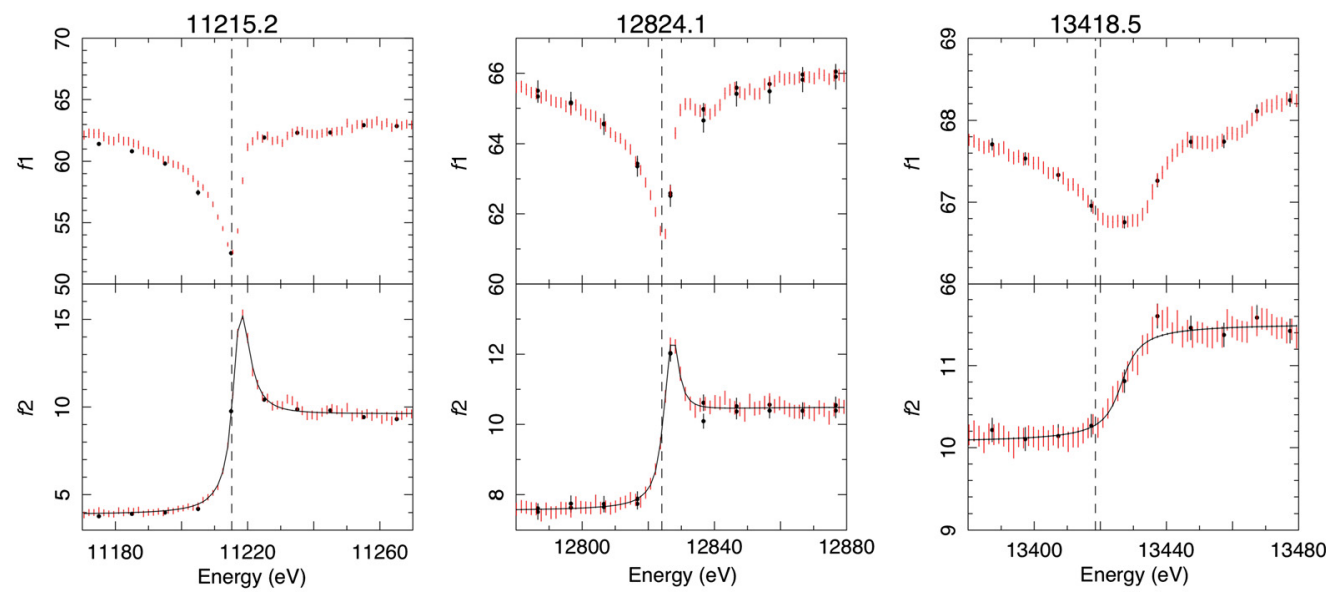

Fig. 14 Atomic scattering factors near the iridium L-edges. The black and red data indicate the results obtained from the coarse and fine pitch scans, respectively. The dashed lines indicate the tabulated energies of the iridium L-edges. ${ }^{18}$ The solid lines present the best-fit Lorentzian + arctan model. The best-fit values are listed in Table 6 . 
Table 6 Best fit parameters of L-edge structure with a Lorentzian and arctan model.

\begin{tabular}{lccc}
\hline \hline & L3 edge & L2 edge & L1 edge \\
\hline Lorentzian & & & - \\
Center energy (eV) & $11217.9 \pm 0.2$ & $12827.3 \pm 0.3$ & - \\
Width (eV) & $6.7 \pm 0.4$ & $4.6 \pm 0.8$ & - \\
Integrated intensity & $20.4 \pm 1.0$ & $8.0 \pm 0.8$ & \\
Arctan & & & $13426.5 \pm 0.6$ \\
Center energy $(\mathrm{eV})$ & $11215.7 \pm 0.3$ & $12824.3 \pm 0.9$ & $28.5 / 62$ \\
\hline$\chi^{2} /$ d.o.f & $36.0 / 59$ & $20.6 / 59$ & \\
\hline \hline
\end{tabular}

Error ranges represent $1 \sigma$ confidence boundaries for a parameter of interest $\left(\chi_{\min }^{2}+1\right)$.

${ }^{a}$ The best fit integrated intensities for $f_{2}$ obtained with the overlayer model are $21.9 \pm 1.0$ for L3 edge and $8.4 \pm$ 0.9 for L2 edge.

\subsection{Comparison with Previous Results and Effects of Overlayer with $\mathrm{f}_{1}$ and $\mathrm{f}_{2}$}

The atomic scattering factors were obtained with an energy pitch of $10 \mathrm{or} 1.5 \mathrm{eV}$ in an energy range of 9 to $15 \mathrm{keV}$ based on the measurement of the reflectivity. The results were compared with the values reported by Henke et al. ${ }^{6}$ As mentioned in Sec. 4.2, the values obtained in this work were slightly smaller than those of Henke et al. ${ }^{6}$ Figure 15 shows the results of the coarse pitch scan data with 0.97 times the values reported by Henke et al. ${ }^{6}$

Graessle et al. ${ }^{8}$ reported the iridium optical constants for the Chandra x-ray mirrors by considering the $\mathrm{x}$-ray reflectance measurements at 0.05 to $12 \mathrm{keV}$. When no overlayer model was used, the angle scan data at $10 \mathrm{keV}$ were represented considering $\beta=2.3106 \times 10^{-6}$, which is $1.2 \%$ smaller than the value of $2.3386 \times 10^{-6}$ used by Henke et al. ${ }^{6}$ When the overlayer model was applied, the optical constant $\beta$ was changed to $2.358 \times 10^{-6}$, whereas $\delta$ remained almost constant $\left(\delta \sim 3.35 \times 10^{-5}\right)$. Massahi et al. (in prep.) pointed out the presence of the overlayer on the SPO plates by considering the calibrations in the lower energy band. Thus, we applied the overlayer model to our angle scan data at the energies of 11,12 , and $14 \mathrm{keV}$. In the employed overlayer model, we assumed a hydrocarbon chain of the form $\mathrm{CH}_{2}$ with a density of $1 \mathrm{~g} / \mathrm{cm}^{3}$,

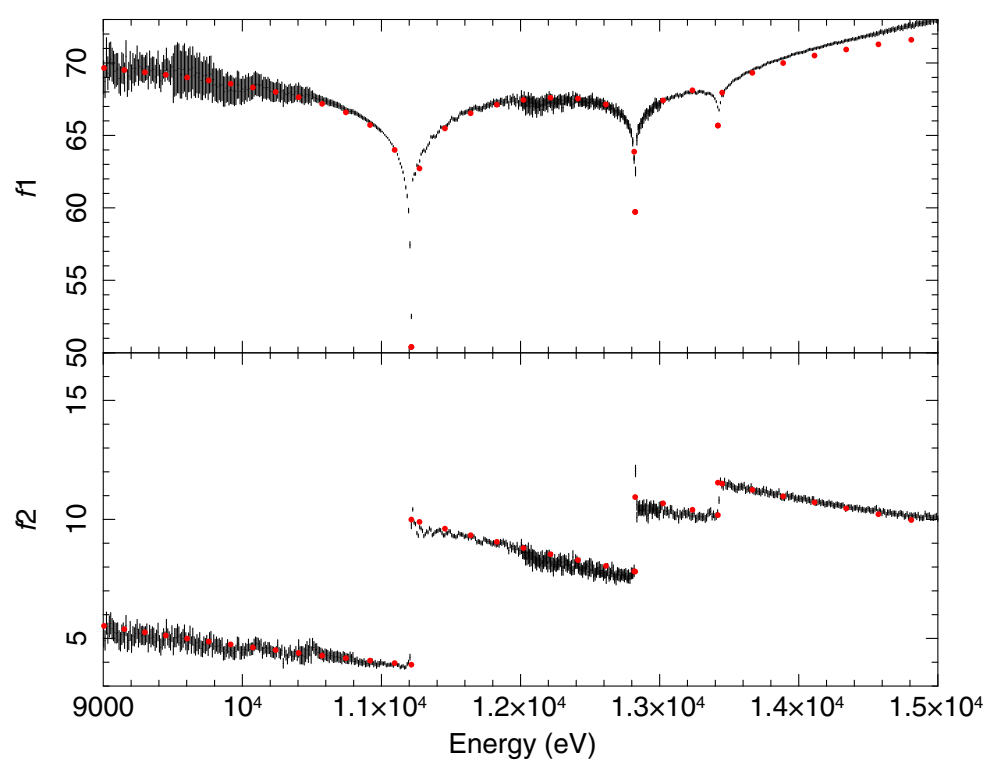

Fig. 15 Derived scattering factors as a function of energy (black) compared to Henke et al.'s values $^{5}$ (red dots) scaled to $97 \%$ to match the scattering factors derived in this work. 
Table 7 Best fit parameters when fitting the angle scan data at $\theta_{I}<1.5$ deg using the overlayer model.

\begin{tabular}{lccc}
\hline \hline & $11 \mathrm{keV}$ & $12 \mathrm{keV}$ & $14 \mathrm{keV}$ \\
\hline Ir $f_{1}$ & $65.60(66.5)$ & $67.71(69.54)$ & $70.58(72.4)$ \\
Ir $f_{2}$ (constant) & 4.13 & 9.07 & 11.2 \\
Thickness $d(\mathrm{~nm})$ & 10.00 & 10.06 & 9.97 \\
Surface roughness $\sigma(\mathrm{nm})$ & 0.230 & 0.25 & 0.00 \\
Interfacial roughness $\sigma_{b}(\mathrm{~nm})$ & 0.647 & 0.661 & 0.630 \\
$\chi^{2} /$ d.o.f. & $392.5 / 64$ & $516.8 / 87$ & $186.0 / 64$ \\
\hline \hline
\end{tabular}

The values reported by Henke et al. ${ }^{6}$ are presented in ( ).

as considered by Graessle et al. ${ }^{8}$ To determine the thickness of the overlayer such that $f_{2}$ matches Henke et al.'s value, ${ }^{6}$ we fitted the angle scan data at $\theta_{I}<1.5$ deg (see Table 7). The best fit surface roughness and overlayer thickness exhibited a scatter when representing the reflectivity above $1 \mathrm{deg}$. Thus, we fitted the data at $\theta_{I}<0.6 \mathrm{deg}$. In the fitting procedure for both the angle and energy scan data, the thickness of the iridium layer was set constant as the mean value $(10.01 \mathrm{~nm})$ obtained using the overlayer model at $\theta_{I}<1.5 \mathrm{deg}$, because it was observed the overlayer thickness was coupled with the thickness of the iridium layer (see Fig. 16). Table 8 lists the best fit parameters obtained using the overlayer model. The angle scan data were well fitted with a small $\chi^{2}$ value.

The fitting to the angle scan data indicated that an overlayer thickness of 0.5 to $0.7 \mathrm{~nm}$ was required to match the best fit $f_{2}$ to Henke et al.'s value. ${ }^{6}$ Subsequently, we applied the overlayer model to the energy scan data and found that an overlayer thickness of $0.9 \mathrm{~nm}$ led to a better fit to the $f_{2}$ data reported by Henke et al. ${ }^{6}$ Figure 17 shows the atomic scattering factors derived from the coarse energy scan. The discrepancy of $f_{2}$ decreased when the overlayer model was applied; however, the discrepancy of $f_{1}$ remained unchanged, as suggested by Graessle et al. ${ }^{8}$ We converted the optical constants $(\delta$ and $\beta)$ at $10 \mathrm{keV}$ measured by Graessle et al. to the atomic scattering factors using Eq. (7) and plotted them in Fig. 17. It is found that our results are consistent

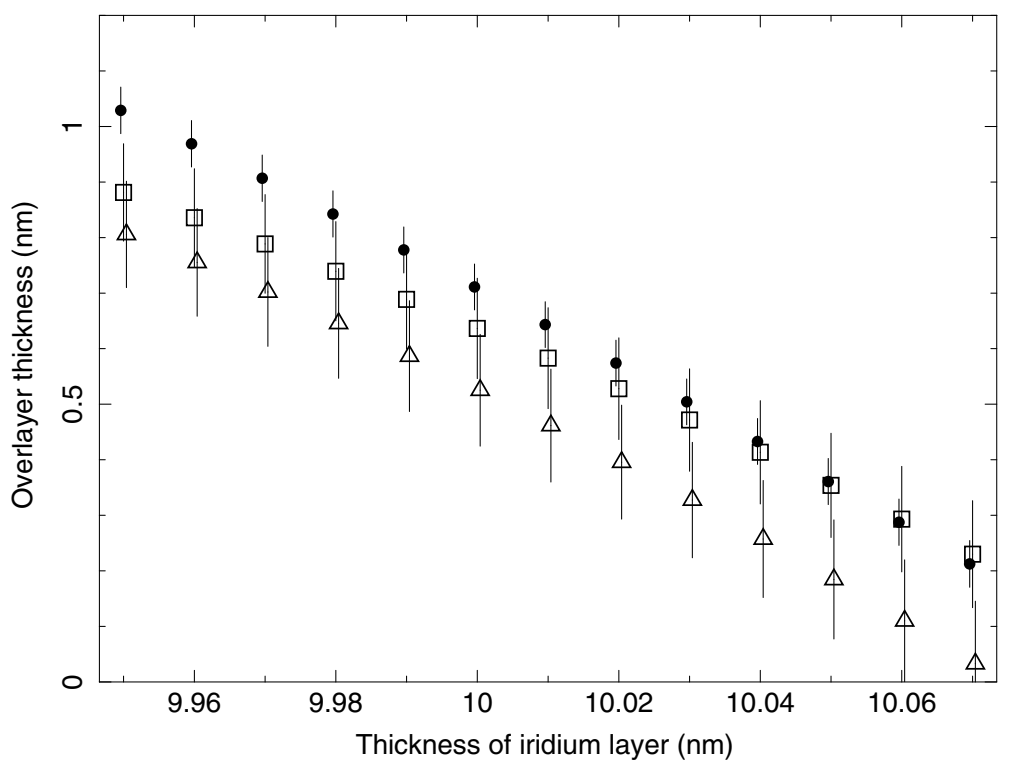

Fig. 16 Dependence of the best fitted overlayer-thickness on the thickness of the iridium layer. The circles, open squares, and open triangles indicate the results for energies of 11,12 , and $14 \mathrm{keV}$, respectively. 
Table 8 Best fit parameters when fitting the angle scan data at $\theta_{I}<0.6$ deg using the overlayer model.

\begin{tabular}{lccc}
\hline \hline & $11 \mathrm{keV}$ & $12 \mathrm{keV}$ & $14 \mathrm{keV}$ \\
\hline Ir $f_{1}$ & $65.67 \pm 0.02$ & $67.91 \pm 0.04$ & $70.87 \pm 0.07$ \\
Ir $f_{2}$ (fixed) & 4.13 & 9.07 & 11.2 \\
Thickness $d(\mathrm{~nm})$ (fixed) & 10.01 & 10.01 & 10.01 \\
Surface roughness $\sigma(\mathrm{nm})$ & $0.296 \pm 0.005$ & $0.319 \pm 0.009$ & $0.307 \pm 0.011$ \\
Interfacial roughness $\sigma_{b}(\mathrm{~nm})$ & $0.643 \pm 0.042$ & $0.583 \pm 0.091$ & $0.462 \pm 0.102$ \\
$\chi^{2} /$ d.o.f & $13.1 / 20$ & $7.6 / 20$ & $6.7 / 20$ \\
\hline \hline
\end{tabular}

Error ranges represent $1 \sigma$ confidence boundaries for a parameter of interest $\left(\chi_{\min }^{2}+1\right)$.

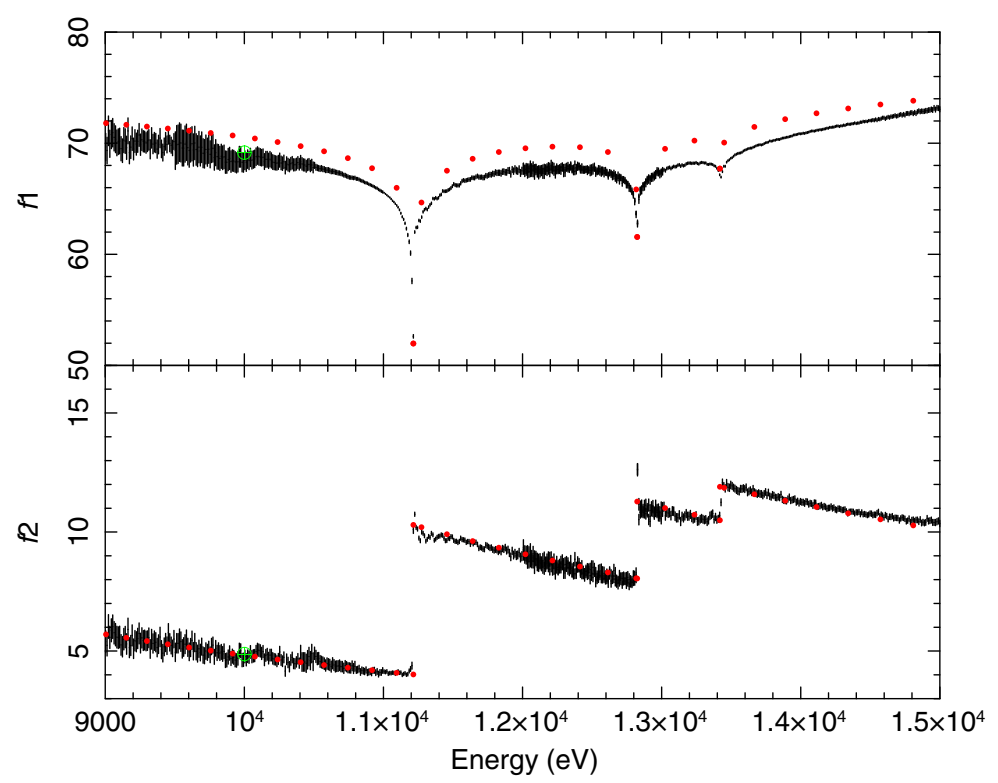

Fig. 17 Atomic scattering factors derived from the overlayer model with a thickness of $0.9 \mathrm{~nm}$. The red marks indicate the atomic scattering factors reported by Henke et al. ${ }^{6}$ The green circles indicate those derived by Graessle et al..$^{8}$ with an overlayer model.

with the results by Graessle et al. The presence of overlayer led to derive the similar $f_{2}$ to the previous results. Henke et al. ${ }^{6}$ adopted the empirical cross section by Biggs and Lighthill ${ }^{7}$ above $10 \mathrm{keV}$ and calculated the atomic scattering factor $f_{1}$ with a Kramers-Kronig dispersion relation. We deduced the $f_{1}$ in the energy region of 9 to $15 \mathrm{keV}$ band from the measurements of the reflectivity.

\section{Conclusions}

We measured the reflectance of an SPO plate coated with an iridium layer with a thickness of $10 \mathrm{~nm}$ at SPring-8 in the energy range covering all iridium L-edges.

1. The angle scan indicated that the surface of the SPO plate was smooth, with a roughness of $0.3 \mathrm{~nm}$, and the thickness of the iridium layer on the SPO substrate was $\sim 10.04 \mathrm{~nm}$., which was consistent with the design value for the considered sample. We confirmed that iridium was successfully deposited on the silicon substrate. 
2. $f_{1}$ and $f_{2}$ near the iridium L-edges were accurately determined, with an uncertainty of only a few percentage points. The energy step of $1.5 \mathrm{eV}$ was less than the energy resolution of the X-IFU. The results can help calculate the effective area of the Athena SPO mirror with a fine energy pitch in the high-energy band.

3. The edge energies of iridium L3 and L2 were consistent with those described in the literature, although the measured edge energy of iridium $\mathrm{L} 1$ was $\sim 8 \mathrm{eV}$ larger than that reported in the literature.

4. Owing to the use of the fine energy pitch, a peak of $f_{2}$ was detected near the iridium L3 and L2 edges. These peaks are usually referred to as XANES.

5. Except for the XANES, the atomic scattering factors were nearly consistent with 0.97 times the values reported by Henke et al. ${ }^{6}$ By considering the existence of the overlayer on the iridium layer, the discrepancies in $f_{2}$ become too small, and the present scattering factor values are consistent with those measured by Graessle et al. The presence of the overlayer is supported by the SPO measurement in the lower energy band. The accumulation of contamination data in future experiments will be important to clarify the impact on performance.

\section{Acknowledgments}

The authors would like to thank anonymous referees for their valuable remarks and suggestions, which have helped us improve this paper. The x-ray measurement was performed at BL20B2 in SPring-8 with the approval of the Japan Synchrotron Radiation Research Institute (JASRI) [Proposal No. 2018B1106 (Y. M.)]. We used the MINUIT software package developed by CERN and a part of the library in HEASOFT developed by NASA/GSFC. This work was financially supported by JSPS KAKENHI through Grant Nos. 17K18782 (H. A.), $15 \mathrm{H02070} \mathrm{(H.} \mathrm{A.),}$ and $20 \mathrm{H} 00175$ (H. M.), and Ehime University (research unit). The authors have no relevant financial interests in the manuscript and no other potential conflicts of interest to disclose.

\section{References}

1. D. Barret et al., "The Athena space X-ray observatory and the astrophysics of hot plasma," Astron. Nachrich. 341, 224-235 (2020).

2. M. Bavdaz et al., "Development of the ATHENA mirror," Proc. SPIE 10699, 106990X (2018)

3. Meidinger, K. Nandra, and M. Plattner, "Development of the wide field imager instrument for ATHENA," Proc. SPIE 10699, 106991F (2018).

4. D. Barret et al. "The ATHENAX-ray integral field unit (X-IFU)," Proc. SPIE 10699, 106991G (2018).

5. H. Wolter, "Spiegelsystem streifenden Einfalls als abbildende Optiken für Röntgenstrahlen," Ann. Phys. 445, 94-114 (1952).

6. B. L. Henke, E. M. Gullikson, and J. C. Davis, "X-ray interactions: photoabsorption, scattering, transmission, and reflection at $E=50-30000 \mathrm{eV}, Z=1-92$," At. Data Nucl. Data. Table 54(2), 181-342 (1993).

7. F. Biggs and R. Lighthill, "Analytical approximations for X-ray cross sections III," Sandia Report, SAND 87-0070 UC-34 (1988).

8. D. E. Graessle et al., "Iridium optical constants for the Chandra X-ray observatory from reflectance measurements of 0.05-12 keV," Proc. SPIE 5165, 469 (2004).

9. N. Kikuchi et al., "Atomic scattering factor of the ASTRO-H (Hitomi) SXT reflector around the gold's L edges," Opt. Exp. 24, 25548 (2016).

10. T. Takahashi et al., "The ASTRO-H (Hitomi) x-ray astronomy satellite," J. Astron. Telesc. Instrum. Syst. 4(2), 021402 (2018).

11. R. L. Kelley et al., "The Astro-H high resolution soft x-ray spectrometer," Proc. SPIE 9905, 99050V (2016).

12. M. E. Eckart et al., "Ground calibration of the Astro-H (Hitomi) soft x-ray spectrometer," J. Astron. Telesc. Instrum. Syst. 4(2), 021406 (2018). 
13. M. J. Collon et al., "Silicon pore optics development for ATHENA," Proc. SPIE 9603, 96030K (2015).

14. B. Landgraf et al., "Development and manufacturing of SPO X-ray mirrors," Proc. SPIE 11119, 111190E (2019).

15. S. Massahi et al., "Installation and commissioning of the silicon pore optics coating facility for the ATHENA mission," Proc. SPIE 11119, 111190F (2019).

16. S. Goto et al., "Construction and commissioning of a 215-m-long beamline at SPring-8," Nucl. Instrum. Methods A 467, 682-685 (2001).

17. M. Yabashi et al., "SPring-8 standard x-ray monochromators," Proc. SPIE 3773, 2-13 (1999).

18. J. A. Bearden and A. F. Burr, "Reevaluation of X-ray atomic energy levels," Rev. Mod. Phys. 39, 125-142 (1967).

19. S. Kraft et al, "High resolution x-ray absorption spectroscopy with absolute energy calibration for the determination of absorption edge energies," Rev. Sci. Instrum. 67, 681 (1996).

20. L. Nevot and P. Croce, "Caractérisation des surfaces par réflexion rasante de rayons X. Application à l'étude du polissage de quelques verres silicates," Rev. Phys. Appl. 15(3), 761-779 (1980).

21. NASA's HEASARC, "HEASoft-A unified release of the FTOOLS and XANADU software packages," https://heasarc.gsfc.nasa.gov/docs/software/heasoft/.

22. D. Girou et al., "Plasma etching for the compatibility of thin film metallic coatings and direct bonding of silicon pore optics," J. Appl. Phys. 128, 095302 (2020).

23. J. H. Sinfelt and G. D. Meitzner, "X-ray absorption edge studies of the electronic structure of metal catalysts," Acc. Chem. Res. 26(1), 1-6 (1993).

24. J. A. Horsley, "Relationship between the area of L2,3 x-ray absorption edge resonances and the d orbital occupancy in compounds of platinum and iridium," J. Chem. Phys. 76, 1451 (1982).

25. V. Monteseguro et al., "Phase stability and electronic structure of iridium metal at the megabar range," Sci. Rep. 9, 8940 (2019).

26. J. A. Bearden, "X-ray wavelengths," Rev. Mod. Phys. 39, 78-124 (1967).

Biographies of the authors are not available. 